\title{
Uso de Indicadores Bióticos Basados en Macroinvertebrados Bentónicos para la Determinación de Calidad Ambiental en la Cuenca Superior del Río Gallegos, Santa Cruz.
}

\section{Bentonic Invertebrates Indexes Used for Determination Environmental Quality Rio Gallegos's Upper Basin. Santa Cruz. Argentina}

\author{
René, ASUETA ${ }^{1}$, reneasueta@hotmail.com \\ Alejandro SÚNICO ${ }^{1}$, alejandrosunico@yahoo.com.mx \\ Juan Pablo MARTIN ${ }^{2}$, martin_jpablo@yahoo.com.ar \\ Carolina SIERPE ${ }^{1}$, clsierpe@gmail.com
}

1. ICASUR, Unidad Académica Río Gallegos, Universidad Nacional de Patagonia. Austral. CAMPUS Universitario Piloto Rivera y Av. Gregores, Río Gallegos, Santa Cruz, Argentina.

2. ICASUR, Unidad Académica San Julián, Universidad Nacional de Patagonia. Colon y Sargento Cabral. Puerto San Julián, Santa Cruz, Argentina.

Recibido: 26/11/2018. Aceptado: 03/04/2019

\begin{abstract}
RESUMEN
La cuenca superior del río Gallegos se encuentra integrada por diferentes cursos de agua, cuya confluencia conforma el río Gallegos. En la cuenca se desarrollan diversas actividades antrópicas, siendo la minería del carbón el principal desarrollo industrial desde 1936. Relacionadas a esta industria se encuentran las localidades de 28 de Noviembre y Río Turbio, cuyos efluentes, tanto urbanos como industriales, son vertidos directamente a los cursos de agua de la cuenca.

En el presente estudio, se analizaron los cambios en la composición de la comunidad de macroinvertebrados bentónicos sujeta a las alteraciones antrópicas y se evaluó la respuesta de distintos índices bióticos en relación con diferentes variables ambientales. Se realizaron cuatro muestreos estacionales de la comunidad de macroinvertebrados bentónicos, se hicieron análisis fisicoquímicos del agua y se aplicaron distintos índices bióticos para determinar el estado ambiental de este sector de la cuenca. En el sitio más cercano a la actividad industrial se detectó un incremento en los valores de Conductividad y DQO, así como también del contenido de hierro soluble en el agua Se detectaron cambios en la comunidad de macroinvertebrados bentónicos, observándose diferencias significativas entre los distintos sitios estudiados. El índice Biotic Monitoring Patagonian Streams (BMPS) mostró la mejor respuesta para evaluar la calidad ambiental del recurso hídrico en el área de estudio. La aplicación de este índice biótico muestra que la calidad de los cursos de agua estudiados varía entre fuertemente contaminados, en cercanías de la actividad industrial minera, y contaminación incipiente en los sitios control y los más alejados de esta actividad.
\end{abstract}

Palabras clave: Calidad de agua; BMPS; Patagonia. 


\begin{abstract}
The Río Gallegos upper basin is integrated by several water courses: San José and Turbio creeks and Rubens and Penitente rivers confluence`s conform the Gallegos. river. Differents anthropic activities are developed in the basin: coal mining, since 1936, is the main historical economic activity. 28 de Noviembre and Río Turbio town`s (population near 10.000 inhabitans) are narrow related with this activity. The urban sewage compound by either domestic or industrial effluents are directly dumped into the water courses without treatment at all. The response of benthic macroinvertebrate communities to human activities was studied extensively in different geographical regions. Meanwhile they had not been neither studied nor used on the water resource in the study area depict the high environmental impact of the activities that take place there.

Different physicochemical analyzes were carried out. The benthic macroinvertebrates community was characterized and different biotic indexes were applied to determine the watercourses environmental's state of this basin's sector. An raise rate in conductivity, iron and QOD values, among others, was detected at the site closest to the industrial activity. This alteration made modifications in the benthic macroinvertebrate community, which presented significant differences between the sites sampled. Diverses biotic indices were used, being the Biotic Monitoring Patagonian Streams (BMPS) the one of them that showed the greatest sensitivity for the determination of the environmental quality of the water resource. The results shown that the watercourses's quality varies between: heavily contaminated in the vicinity of industrial activity, and incipient contamination in places furthest away from these activities mainly beyond the river. confluence mentioned. The main environmental impact is caused by urban sewage and mining activity.
\end{abstract}

Key words: Water quality; BMPS; Patagonia.

\title{
1. INTRODUCCIÓN
}

La denominada Cuenca del río Gallegos $\left(51^{\circ} 32^{\prime} 08^{\prime \prime} \mathrm{S}-72^{\circ} 20^{\prime} 08^{\prime \prime} \mathrm{O}\right)$ se ubica al sur de la provincia de Santa Cruz, Argentina (Ilustración 1). Se localiza en el Departamento de Güer Aike e incluye las localidades de Río Turbio y 28 de Noviembre y los parajes de Julia Dufour, Turbio Viejo y Rospentek. Esta región constituye en gran parte la cabecera de la cuenca del río Gallegos, el principal curso de agua del sur de la provincia.

La cuenca hidrográfica del río Gallegos tiene una extensión de $300 \mathrm{~km}$ y abarca alrededor de $8400 \mathrm{Km}^{2}$ (Caballero 2000) y se origina a partir de los arroyos Santa Flavia y San José, y los ríos Turbio, Penitentes y Rubens. Aguas abajo de las localidades mencionadas se ubican otros parajes como Puente Blanco y Bella Vista entre otros y ya en la desembocadura del río Gallegos, la ciudad homónima, capital de la Provincia de Santa Cruz. 


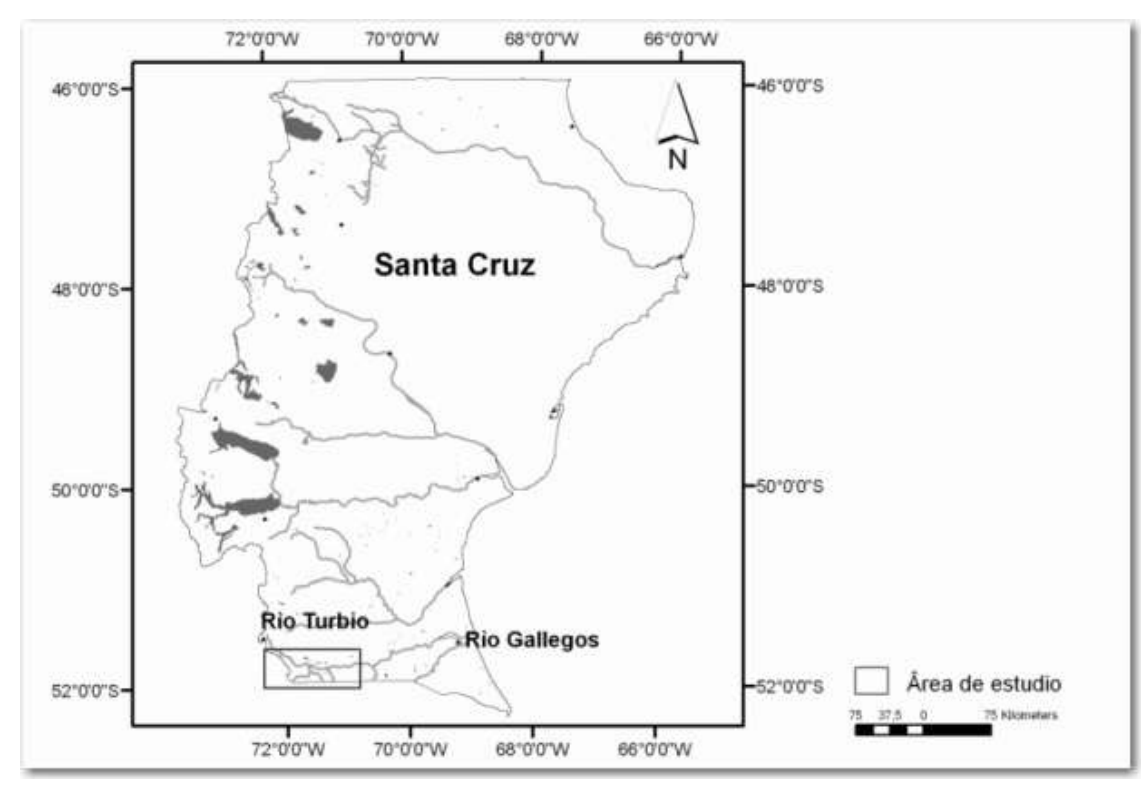

Ilustración 1: Sitio y posición del área de estudio

Los arroyos Santa Flavia y San José atraviesan el ejido urbano de la localidad de Río Turbio, motivo por el cual, tanto sus valles y cauces como los atributos de sus aguas, se hallan fuertemente disturbados, ya sea por modificaciones realizadas a sus cauces (ejemplo más notorio el dique San José) o por el aporte de efluentes urbanos e industriales (Esteves et al. 1996, Caballero 2009 a y b, Asueta et al. 2013) y/o de material estéril proveniente de la actividad minera del carbón que se desarrolla en el sector. Estos arroyos aportan sus aguas al río Turbio, el cual recorre todo el sector de disposición de estériles de mina y transcurre aledaño al sitio de instalación de la Usina Termoeléctrica Río Turbio. Éste también atraviesa el ejido urbano de la localidad de 28 de Noviembre, donde se registran nuevos aportes de efluentes provenientes de pluviales y cloacas. Recorre alrededor de $120 \mathrm{~km}$ antes de confluir con el río Gallegos, del cual se abastece de agua potable a la ciudad homónima.

El uso de macroinvertebrados como indicadores de la calidad de las agua y de los ecosistemas acuáticos ha demostrado ser de gran utilidad, generalizándose su uso tanto con fines científicos como aplicados (Prat et al. 008). Su relación con el impacto de las actividades humanas, en particular las urbanas e industriales, ha sido profusamente estudiada. Entre estos estudios se encuentran numerosos orientados a determinar como la sostenida explotación de una mina de carbón puede condicionar su comportamiento y existencia. El yacimiento carbonífero Río Turbio, en explotación desde alrededor de 1939 (Zoccola 1973), nos brinda una excelente oportunidad para estudiar esta relación en la Patagonia austral.

Los objetivos del presente trabajo fueron analizar los cambios en la composición de la comunidad de macroinvertebrados bentónicos de la cuenca superior del río Gallegos sujeta a alteraciones antrópicas y analizar la respuesta de distintos índices bióticos en relación a diferentes variables ambientales. Los índices bióticos que mejor reflejan la calidad ambiental de la cuenca podrán ser propuestos como herramientas de gestión tendientes a alcanzar la sustentabilidad ambiental del recurso hídrico estudiado.

\section{MARCO CONCEPTUAL}

En las últimas décadas, los sistemas fluviales en el mundo han estado sometidos a una fuerte presión de explotación y a cambios en el uso de la tierra, que afectaron la calidad de sus aguas (Branco 1984, Citado en Gamboa et al. 2008). La Cuenca Superior del río Gallegos no se 
exceptúa de esta situación, encontrándose bajo una fuerte presión antrópica en toda su extensión desde fines de la década 1890, presión que se intensificó con el desarrollo de la mina de carbón de Río Turbio desde 1936.

Las alteraciones producto de la actividad humana en los ambientes lóticos implican modificaciones en sus propiedades físicas y químicas y de la estructura de su biocenosis (Welch y Lindell 1992). Los cambios en el uso de la tierra son considerados el factor de stress más importante para los ecosistemas fluviales (Carpenter 1992, Citado por Bruns 2005), cuyos impactos más relevantes se hallan asociados a la modificación de las cuencas hidrográficas y a la contaminación de los recursos acuáticos producto de las diferentes actividades humanas (Bruns 2005).

En la actualidad, son numerosas las causas que provocan la degradación del agua y de las comunidades biológicas que habitan en ella. Entre las principales causas se encuentran: la contaminación por materia orgánica y el enriquecimiento en nutrientes (vertido de efluentes cloacales), la eliminación o degradación de los bosques (incremento de la carga de sedimentos), la rectificación y canalización de los ríos (pérdida de caudal y salinización), la regulación de cauces y la construcción de represas y centrales hidroeléctricas, la presencia de contaminantes inorgánicos y orgánicos persistentes o las actividades mineras, petroleras e industriales (Prat y Ward 1994, Allan 1995, Wetzel 2001, Angelier 2002, Suárez et al. 2002, Toro et al. 2002. Citados en Alonso y Camargo 2005).

La minería es una actividad que generalmente se acepta como altamente impactante sobre los recursos hídricos. Las descargas de los efluentes de las actividades mineras pueden generar un severo empobrecimiento de la flora y fauna de los ríos aguas abajo de los puntos de descarga (Armitage y Blackburn 1985 Citado en Batty et al. 2005, Jarvis y Younger 1997, Malmqvist y Hoffsten 1999 Citado en Batty et al. 2005.). Estos efluentes pueden ser causados por la inundación de las labores subterráneas y o a cielo abierto, en el caso de minas abandonadas, lixiviación de escombreras, relaves del mineral de reserva (Banks et al. 1997 Citado en Batty et al. 2005, Younguer 1997 Citado en Batty et al. 2005, Manzano et al. 1991 Citado en Manzano et al. 1999.), filtraciones y lixiviados de diques de colas.

Es común la asociación del carbón y la roca de caja, dado el ambiente geológico de formación del carbón, con sulfuros de hierro, en particular el mineral Pirita $\left(\mathrm{FeS}_{2}\right)$. Esta asociación genera un problema ambiental que frecuentemente afecta a estos yacimientos denominado drenaje ácido de mina (DAM), producido tanto en las acumulaciones de estériles como en los drenajes de agua de mina. El DAM se inicia con los ciclos de humectación y desecación de la Pirita expuesta a los agentes atmosféricos, liberándose ácido sulfúrico al ambiente. De este modo, se da inicio a una serie de reacciones que pueden generar nuevos compuestos así como solubilizar elementos, en particular metales. Dependiendo de la composición del estéril, se pueden generar reacciones de neutralización del ácido generado y la liberación de $\mathrm{Na}, \mathrm{Ca}, \mathrm{Si}, \mathrm{K}$ y Al, elementos muy comunes en la Cuenca Carbonífera dada las características litológicas de las sedimentitas acompañantes de los mantos de carbón. La composición del DAM será específica de cada lugar. La velocidad de producción ácida es controlada por factores como la temperatura, $\mathrm{pH}$ y fuerza electroquímica de la solución de contacto, mineralogía de los estériles o roca de caja, área superficial de las partículas de sulfuro y la actividad biológica (Van Huyssteen 1998).

\section{El uso de macroinvertebrados bentónicos como bioindicadores}

Los primeros intentos de evaluar las alteraciones producidas en el medio acuático por la actividad humana se basaron en una valoración fisicoquímica de la calidad del agua, estableciendo umbrales de concentración para algunas sustancias consideradas tóxicas o indicadoras de calidad (Alonso y Camargo 2005). Sin embargo, estos umbrales, en general se 
referenciaban a estándares internacionales para algún fin específico (recreación, consumo humano, consumo animal, etc.). No se evaluaba, sin embrago, cómo la variación en las características ambientales naturales u originales afectaban al ecosistema en general y a los organismos en particular, que utilizan estos ambientes para el desarrollo de todo o parte de su ciclo vital.

Una solución integral para evaluar el impacto sobre un ecosistema consiste en estudiar una comunidad biológica, ya que su estructura funcional integra el efecto de muchos factores ambientales $\mathrm{y}$, además, necesita un tiempo más o menos prolongado para recuperarse tras sufrir una perturbación (Alonso et al. 2005).

El uso de bioindicadores consiste en la medición de variables biológicas para estudiar el medio ambiente. En este sentido, un bioindicador puede estar dado por alguna característica particular en algún nivel de organización, que va desde el suborganismo (gen, célula, tejido) o los niveles de organismo, población, comunidad o inclusive ecosistemas (Niemi y McDonald 2004 Citado en Bonada et al. 2006).

El monitoreo de los cambios en la calidad de agua en ríos y arroyos se puede realizar mediante el estudio de los organismos acuáticos. La biota residente en un cuerpo de agua es un indicador natural de la calidad ambiental y puede revelar los efectos de la alteración del hábitat. Los organismos bentónicos son los más utilizados como bioindicadores de calidad de agua en ríos (Miserendino et al. 1999).

En la Argentina, el estudio del comportamiento de los maroinvertebrados bentónicos de agua dulce, en relación a la presión ejercida sobre ellos por diferentes actividades antrópicas, se encuentra bien desarrollado, pudiendo mencionarse a autores como: Miserendino (2000, 2001 y 2009), Miserendino y Pizzolón (1992, 1999), Miserendino, Brand y Prinizio (2008), Miserendino y Brand (2009), Rodrigues Capítulo, Tangorra y Ocón (2001), Fernández y Domínguez (2001), Domínguez y Fernández (2009), Tripole y Corigliano (2005) entre otros. En la provincia de Santa Cruz existe un escaso desarrollo de este tipo de estudios y poca información que haya sido publicada. En la actualidad, se encuentran publicaciones referidas a las cuencas de los ríos Santa Cruz, Chico y Deseado (Miserendino, 2001). Por otro lado, existen estudios de línea de base previa a la construcción de las represas Néstor Kirchner y Jorge Cepernic en donde Tagliaferro et al., (2013) realizan una caracterización de la comunidad de macroinvertebrados bentónicos del río Santa Cruz. Martin et al. (2014) plantea también para el río Santa Cruz, la necesidad de establecer índices bióticos para poder evaluar y monitorear los posibles impactos que se generen a partir de la construcción de las represas. No se encontraron trabajos de similares características sobre la cuenca del río Gallegos en toda su extensión

El progresivo deterioro producido en los ecosistemas acuáticos por las modernas sociedades hace necesaria la detección rápida de los cambios en la calidad del agua (Miserendino y Pizzolón 1999). Los índices bióticos son herramientas de valoración de la calidad ambiental basados en las diferentes respuestas de los organismos a las alteraciones del medio (grado de sensibilidad o tolerancia) (Confederación Hidrográfica del Ebro, 2005). Estos índices son una de las maneras más comunes de establecer la calidad ambiental de los ríos, y habitualmente consisten en la combinación de dos o tres propiedades de la comunidad, como la riqueza de taxa, la abundancia y la tolerancia/intolerancia de los diferentes taxa a la contaminación (Prat et al. 2008).

Los índices bióticos que utilizan macroinvertebrados bentónicos de agua dulce más desarrollados actualmente son los basados en el método BMWP (Biological Monitoring Working Party). Este índice combina el número de taxa con un valor de tolerancia/intolerancia de los diferentes taxa a nivel de familia. El valor final se obtiene de una sumatoria de los valores de tolerancia/intolerancia de cada una de las familias, que van desde 0 a 10. La adaptación de estos índices a determinadas regiones en América del Sur ha 
originado la creación de otros, tales como el Índice Patagónico Andino (IAP) y el Biological Monitoring Patagonian Streams (BMPS) (Miserendino et al. 1992, 1999) diseñados para evaluar la contaminación orgánica en el norte de la región Patagónica. Igualmente los índices BMWP y ASPT fueron utilizados para los ríos de montaña de Tucumán y otras provincias del noroeste de Argentina (Domínguez et al. 2009).

\section{MATERIALES Y MÉTODOS}

\section{1 Área de estudio}

La realización de un relevamiento exploratorio en el área de estudio permitió definir 8 sitios de muestreo dentro de la cuenca hídrica superior del río Gallegos. Estos sitios fueron distribuidos aguas arriba y aguas abajo de las zonas más afectadas por la actividad minera actualmente en desarrollo, hasta llegar a la unión del río Gallegos con el río Turbio (Ilustración 2).

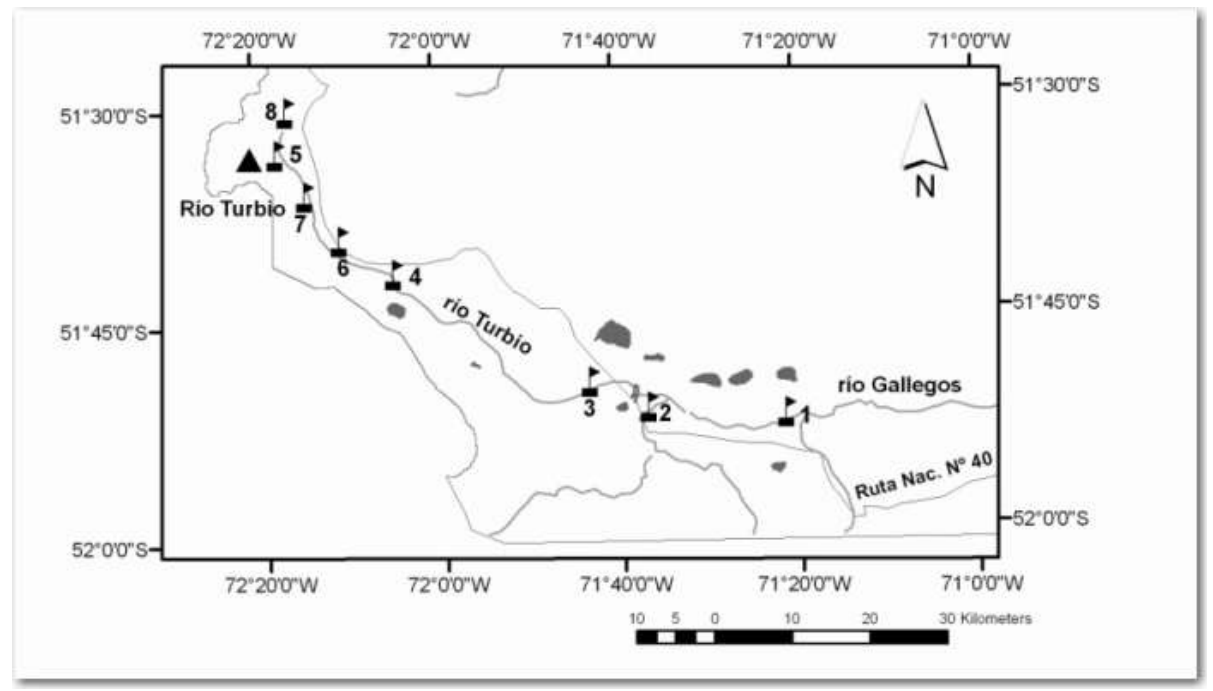

Ilustración 2: Ubicación de los sitios de muestreo en la cuenca superior del Río Gallegos.

Sitio 1 (Morro Chico): Es el sitio aguas abajo más alejado de la actividad minera. En este sector se desarrolla cría de ganado y actividades recreativas como la pesca deportiva.

Sitio 2 (Puente Blanco): Este sitio se ubica en el tramo inicial del río Gallegos y se utiliza como un sector de acampe y esparcimiento. Además se encuentran instalaciones de dependencias administrativas Provinciales y Nacionales.

Sitio 3 (Glen Cross): este sitio es el último ubicado sobre el río Turbio y es el más alejado de la actividad minera. En este sector de la cuenca, la actividad principal es la cría de ganado bovino y ovino.

Sitio 4 (Turbio Viejo): este sitio se encuentra en cercanías del paraje Turbio Viejo, donde funcionan dependencias administrativas Provinciales, Nacionales y el desarrollo de pequeñas chacras.

Sitio 5 (Frente a Mina): Este punto se encuentra frente al complejo industrial minero. Se ubica en el tramo final del arroyo San José y se encuentra afectado por varias actividades, como ser la actividad minera propiamente dicha, la planta de tratamiento de efluentes cloacales de la localidad de Río Turbio y la actividad de una cantera.

Sitio 6 (Entre 28 y Rospentek): Este sitio de muestreo se encuentra sobre el río Turbio entre la localidad de 28 de Noviembre y el paraje Rospentek, la actividad principal es la ganadería de ovinos y vacunos. 
Sitio 7 (El Mirador): Este sitio se encuentra en zona de chacras de la Localidad de 28 de Noviembre, en cercanías de la base del Mirador de Cóndores de la Localidad.

Sitio 8 ( $\mathbf{A}^{\circ}$ Primavera): este sitio se encuentra en el Valle Primavera, un sector del río Turbio que la comunidad de la Cuenca Carbonífera utiliza para el esparcimiento. Se encuentra ubicado aguas arriba del complejo minero, y fue considerado como un sitio control o de referencia con bajo impacto antrópico.

\subsection{Metodología de muestreo}

En cada sitio se realizaron cuatro muestreos estacionales, tanto de macroinvertebrados bentónicos como de agua para análisis fisicoquímicos.

Las muestras para análisis fisicoquímicos fueron tomadas manualmente en botellas de un litro de capacidad color caramelo, con tapa hermética, sin cámara de aire y con el agregado de ácido nítrico, en el caso de las muestras de metales, para garantizar su conservación. Para su traslado se dispuso de contenedores con aislante, para mantener durante el transporte una temperatura no mayor de $4^{\circ} \mathrm{C}$ hasta la llegada al laboratorio (Norma IRAM 29012-3). Los parámetros evaluados fueron pH (Método ASTM D 1293-84), Turbidez (turb) (Norma IRAM 29011), Conductividad (cond) (Norma ASTM D 1193-91), Sólidos Disueltos Totales (St) (Norma ISO 9562(1989) Método AOX-DIN/38409-H14), Sólidos Sedimentables en 10min y 2hs (Norma ISO 11923:97), Oxígeno Disuelto (OD) (Norma IRAM 29008), Demanda Química de Oxigeno (DQO) (Norma IRAM 29007-1), Demanda Biológica de Oxígeno (DBO) (Norma IRAM 29007-2), Metales (Normas IRAM NM 300-6:2003 e IRAM 301 Norma ISO/IEC 17025).

En cada sitio se tomaron cuatro muestras de la comunidad de macroinvertebrados bentónicos utilizando una red manual con boca de $50 \times 50 \mathrm{~cm}$ y malla de $500 \mu \mathrm{m}$, y un marco metálico para delimitar un área de muestreo de $0,25 \mathrm{~m}^{2}$ sobre el fondo. Las muestras se tomaron en una transecta paralela a la margen del río entre 30 y $40 \mathrm{~cm}$ de profundidad. El sustrato fue removido con pala durante 2 minutos y las rocas de mayor tamaño fueron lavadas en forma manual en la boca de la red. Las muestras se fijaron in situ con solución de formol al $5 \%$.

En el laboratorio, se separaron los organismos bajo lupa estereoscópica, se identificaron a nivel de familia o del nivel taxonómico más bajo posible utilizando guías regionales Fernández et al. (2001). Luego de su identificación los organismos se contabilizaron y se guardaron en alcohol $70 \%$ para su preservación.

\subsection{Análisis de datos}

Los datos fisicoquímicos y biológicos obtenidos fueron analizados y comparados estadísticamente entre sitios de muestreo y entre estaciones del año mediante análisis univariados y multivariados, utilizando los paquetes estadísticos Infostat versión 2016p y PRIMER 6.1.

Para cada muestra de macrobentos se calculó la abundancia total y relativa (\%) de cada taxón; la riqueza de taxones $(\mathrm{S})$, definida como el número de taxones presentes en la muestra, y la diversidad ( $\left.\mathrm{H}^{\prime}\right)$ utilizando el índice de diversidad de Shannon-Wiener.

Los datos de abundancia fueron analizados mediante técnicas de agrupamiento (Hierarchical agglomerative clustering, HAC) y de ordenamiento no paramétrico (Multidimensional Scaling, MDS). Los datos fueron previamente transformados utilizando $\log (\mathrm{x}+1)$ y posteriormente se aplicó el Índice de Similitud de Bray-Curtis. La significancia estadística de los agrupamientos entre muestras fue corroborada mediante el test de permutación ANOSIM, para poner a prueba la hipótesis de diferencias en la composición de la comunidad entre épocas y entre sitios de muestreo. La rutina SIMPER fue utilizada para identificar a los taxones que caracterizaron a cada agrupamiento de muestras (Clarke y Warwick 2001). 
Se calcularon para cada muestra los siguientes índices bióticos indicadores de calidad ambiental:

- EPT: Se basa en los Órdenes de insectos Ephemeroptera, Plecoptera y Trichoptera, uno de los más sensibles a los cambios ambientales. Se calcula como:

$$
E P T=(\text { Ab. EPT } / \text { Ab. total }) \times 100
$$

donde Ab. EPT es el número de individuos de Ephemeroptera, Plecoptera y Trichoptera en la muestra, y Ab. total es el número de individuos de todos los taxa presentes en la muestra (Gray y Delaney, 2007).

- EPTCh: Es una variante del anterior, que incluye la abundancia de Chironomidae (Diptera), uno de los grupos más tolerantes a los disturbios ambientales. Se calcula como:

$$
E P T C h=(\text { Ab. EPT / Ab. EPT }+ \text { Ab. Chironomidae }) \times 100
$$

donde Ab. EPT es el número de individuos de Ephemeroptera, Plecoptera y Trichoptera en la muestra, y Ab. Chironomidae es el número total de individuos de Chironomidae en la muestra (Gray et al. 2007).

-BMPS (Biotic Monitoring Patagonian Streams), índice biótico a nivel de familias de macroinvertebrados propuesto por Miserendino et al. (1999), para los cursos de agua andino patagónicos. A cada familia se le asigna un puntaje entre 1 y 10 de acuerdo a su sensibilidad a los disturbios ambientales, siendo 10 el puntaje para las familias más sensibles y 1 para las más tolerantes. El índice se calcula sumando el puntaje de todas las familias presentes en la muestra.

- ASPT: adaptado del Average Score Per Taxa, se calculó en este trabajo como:

$$
A S P T=B M P S / \mathrm{N}
$$

donde $\mathrm{N}$ es el número total de familias utilizado en el cálculo del BMPS.

\section{RESULTADOS}

\subsection{Caracterización fisicoquímica}

Los parámetros ambientales que mostraron mayor diferencia entre sitios se presentan en las gráficas realizadas para las cuatro estaciones del año (Figura 1). El Sitio 5 mostró claros incrementos en los valores de conductividad, sólidos totales, cloruros, dureza, fosfatos, turbidez, DBO, DQO y concentración de hierro en todas las épocas del año, en particular si lo comparamos con los Sitios 1 y 8 . Como se describiera anteriormente, estos dos últimos sitios representan las condiciones más similares al ambiente natural y menos antropizado. El primero por hallarse más lejano aguas abajo respecto a las actividades impactantes, y posterior a la confluencia de los ríos Rubens y Penitente que con su importante caudal podrían estar diluyendo cualquier aporte proveniente de aguas arriba; el segundo por estar aguas arriba del sitio de mayor disturbio y en una subcuenca que no se halla afectada por las actividades descriptas. 


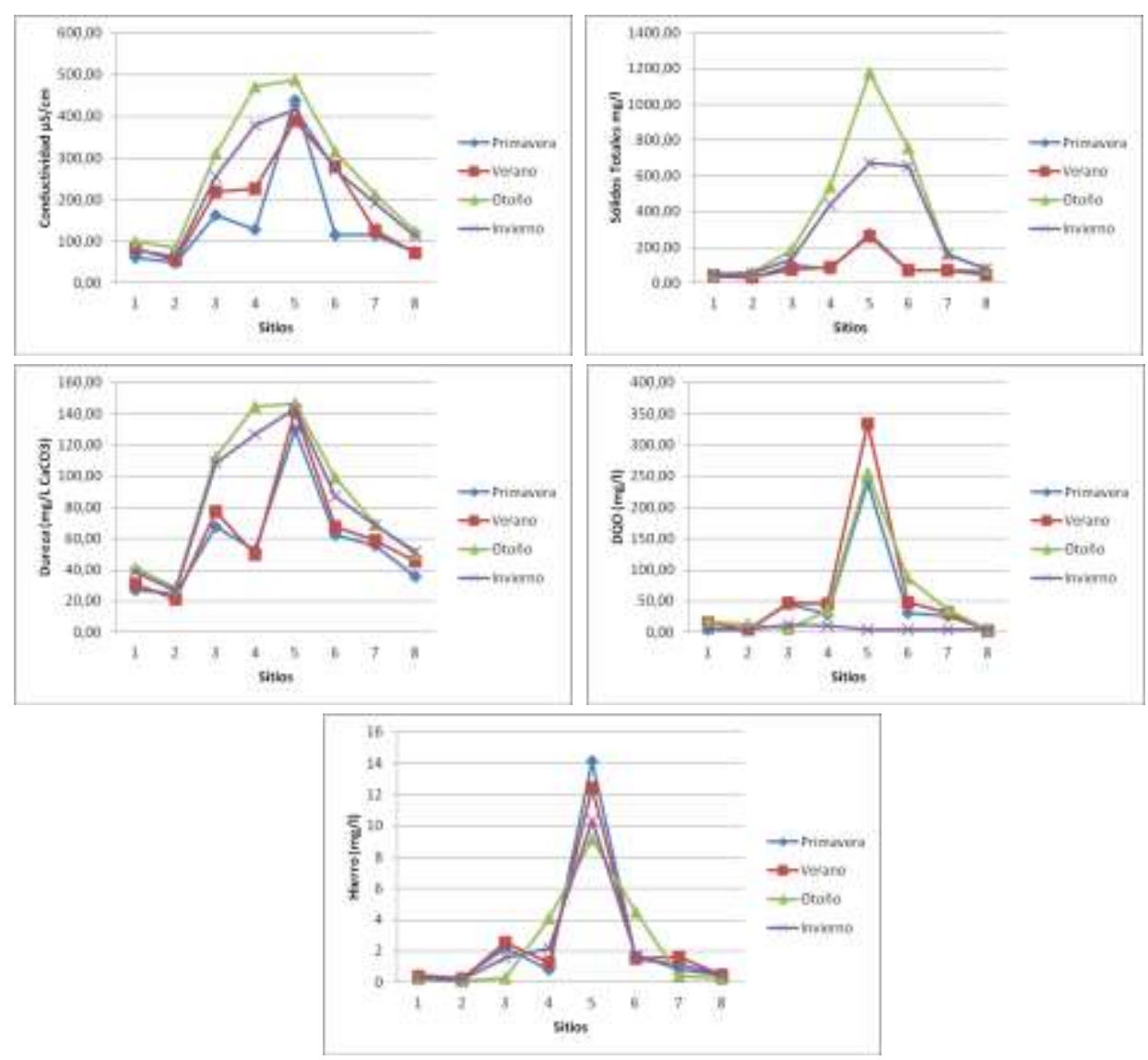

Figura 1: Variación de los parámetros fisicoquímicos entre los diferentes sitios de muestreo para las estaciones del año muestreadas

\subsection{Comunidad de macroinvertebrados bentónicos}

La comunidad de macroinvertebrados estuvo integrada por 22 taxa, siendo los mejor representados los nematodes, los anélidos oligoquetos e hirudíneos, los moluscos gasterópodos del género Lymnaea (familia Lymnaeidae), los crustáceos anfípodos Hyalella sp. (familia Dogielinotidae) y diferentes familias de insectos. Entre estos últimos se destacaron por su abundancia los dípteros de la familia Chironomidae, los efemerópteros de la familia Baetidae, los plecópteros Perlidae, los tricópteros Glossosomatidae y los coleópteros Elmidae.

Se observaron diferencias en la composición de la comunidad a lo largo de la cuenca y una marcada disminución de taxa en el Sitio 5, donde la comunidad se encontró marcadamente dominada por oligoquetos (Tabla 1). Los sitios 1, 2 y 8 mostraron una mayor riqueza de taxa en la comunidad, entre los que se pueden destacar por su abundancia los anfípodos Hyalella y diferentes familias de insectos (Baetidae, Perlidae, entre otros). Salvo los sitios 1 y 8 , el resto de los sitios mostraron una proporción mayor de oligoquetos en la comunidad. 
Tabla 1: Abundancia promedio de los diferentes taxa de macroinvertebrados bentónicos en cada uno de los sitios de muestreo.

\begin{tabular}{|c|c|c|c|c|c|c|c|c|}
\hline & Sitio 1 & Sitio 2 & Sitio 3 & Sitio 4 & Sitio 5 & Sitio 6 & Sitio 7 & Sitio 8 \\
\hline Nematoda & 1 & 3 & 0 & 1 & 42 & 2 & 3 & 1 \\
\hline Oligochaeta (Annel.) & 72 & 336 & 147 & 1958 & 481 & 2458 & 496 & 98 \\
\hline Glossiphoniidae (Annel. Hirud.) & 5 & 3 & 2 & 41 & 1 & 36 & 4 & 6 \\
\hline Lymnaeidae (Mol. Gast.) & 65 & 4 & 0 & 30 & 0 & 0 & 0 & 0 \\
\hline Sphaeriidae (Mol. Biv.) & 0 & 0 & 0 & 0 & 0 & 0 & 0 & 0 \\
\hline Dogielinotidae (Crust. Amph.) & 125 & 73 & 70 & 293 & 2 & 9 & 1 & 39 \\
\hline Ostracoda (Crust.) & 1 & 0 & 3 & 30 & 36 & 22 & 20 & 0 \\
\hline Glossosomatidae (Trichop.) & 2 & 3 & 0 & 11 & 2 & 14 & 6 & 2 \\
\hline Perlidae (Plecop.) & 39 & 17 & 24 & 1 & 1 & 1 & 0 & 139 \\
\hline Baetidae (Ephemerop.) & 63 & 52 & 0 & 0 & 0 & 0 & 1 & 70 \\
\hline Simuliidae (Dip.) & 14 & 3 & 0 & 0 & 0 & 0 & 0 & 1 \\
\hline Culicidae (Dip.) & 0 & 0 & 0 & 0 & 0 & 0 & 0 & 0 \\
\hline Chironomidae (Dip.) & 16 & 31 & 13 & 197 & 20 & 148 & 61 & 191 \\
\hline Tipulidae (Dip.) & 1 & 0 & 0 & 0 & 0 & 0 & 0 & 0 \\
\hline Tabanidae (Dip.) & 0 & 0 & 0 & 0 & 0 & 0 & 0 & 0 \\
\hline Muscidae (Dip.) & 0 & 0 & 0 & 0 & 0 & 0 & 0 & 0 \\
\hline Mesoveliidae (Hemip.) & 0 & 0 & 1 & 4 & 1 & 2 & 1 & 1 \\
\hline Elmidae (Coleop.) & 25 & 9 & 0 & 0 & 0 & 0 & 3 & 2 \\
\hline Hydrophilidae (Coleop.) & 1 & 1 & 0 & 0 & 0 & 0 & 0 & 1 \\
\hline Odonata & 0 & 1 & 0 & 1 & 0 & 0 & 0 & 0 \\
\hline Hydrachnidae (Acari) & 1 & 1 & 0 & 2 & 0 & 1 & 0 & 4 \\
\hline
\end{tabular}

Los análisis multivariados realizados mostraron que no existieron diferencias estacionales significativas en la composición de la comunidad, sin embargo, demostraron importantes diferencias espaciales entre los diferentes sitios de muestreo. Los análisis de ordenamiento (MDS) y agrupamiento (HAC) (Figura 3) aplicados al muestreo de primavera, permitieron identificar cinco grupos principales de muestras, coincidentes entre ambos análisis (ANOSIM: $\mathrm{R}$ global $=0,837, \mathrm{P}=0,001)$. 


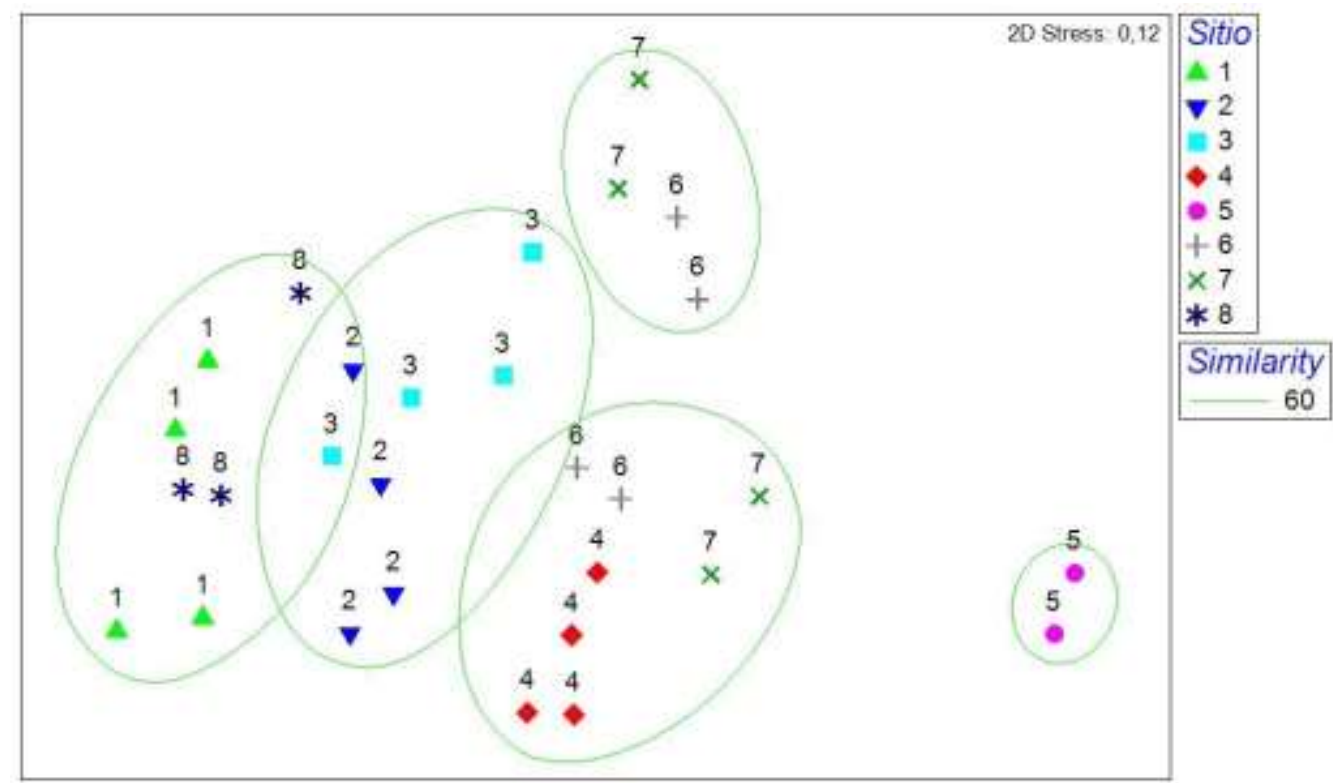

Figura 3: Análisis de Ordenamiento (MDS) y agrupamiento (HAC) elaborado para primavera en función de las muestra de macroinvertebrados por sitios de muestreo.

En la estación de verano, los análisis de ordenamiento y agrupamiento (Figura 4), permitieron identificar cuatro grupos principales de muestras, coincidentes entre ambos análisis, según la similitud en la composición de la comunidad (ANOSIM: $\mathrm{R}$ global $=0,898, \mathrm{P}=0,001$ ).

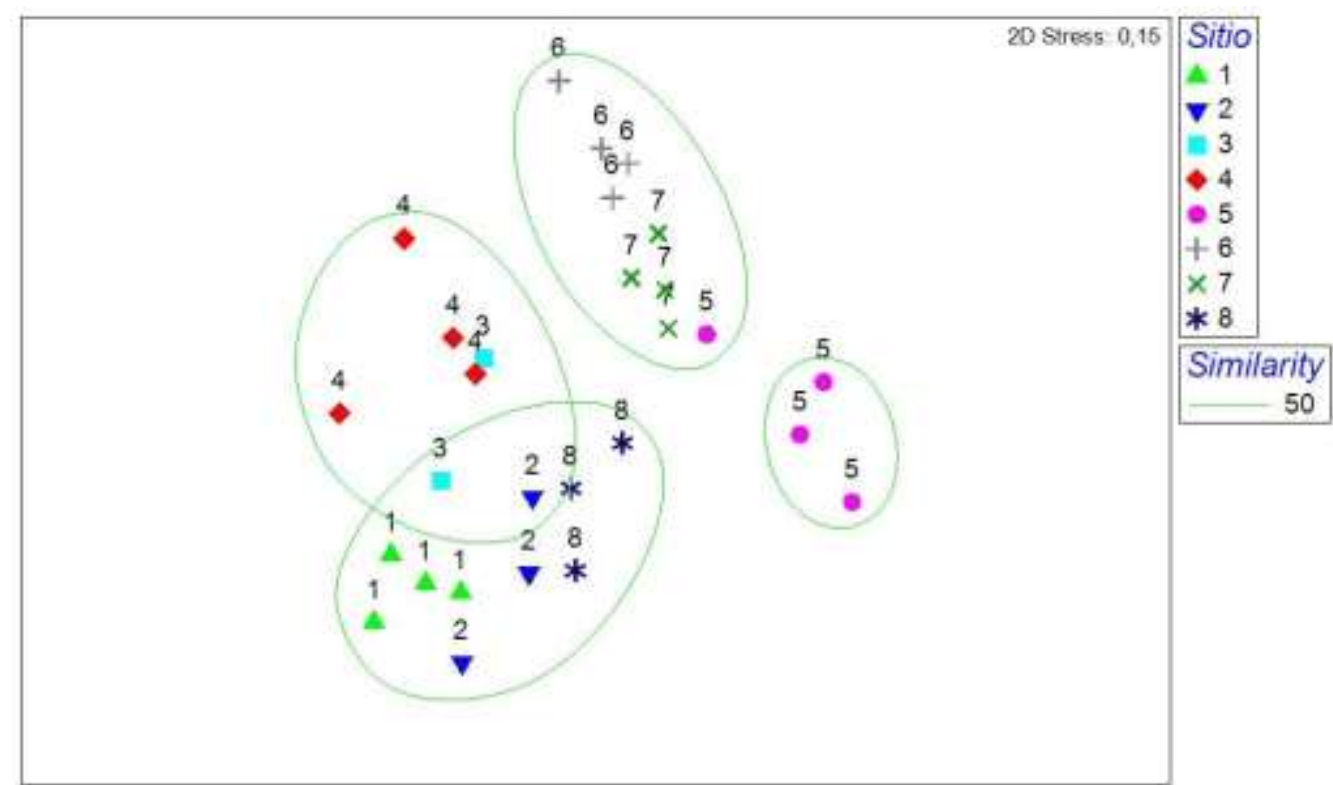

Figura 4: Análisis de Ordenamiento (MDS) y agrupamiento (HAC) elaborado para el muestreo de verano en función de las muestras de macroinvertebrados por sitios de muestreo.

En ambas épocas del año, las muestras del sitio 5 conformaron un único grupo separado de las muestras de los demás sitios. Las muestras correspondientes a los sitios 1 y 8 se agruparon juntas en ambas épocas del año.

El análisis SIMPER permitió identificar a los taxa que caracterizaron a la comunidad en los diferentes agrupamientos de muestras para cada época del año analizada (Tablas 2 y 3). 
Tabla 2: Análisis SIMPER. Principales taxa que contribuyen a la similitud de muestras dentro de cada grupo en primavera. $\mathrm{AP}=$ abundancia promedio (datos transformados); $\mathrm{SP}=$ similitud promedio; \% $\mathrm{C}=$ porcentaje de contribución; \% AC=porcentaje acumulado de contribución.

\begin{tabular}{|c|c|c|c|c|c|}
\hline & Taxa & $\mathbf{A P}$ & SP & $\% \mathrm{C}$ & $\% \mathrm{AC}$ \\
\hline Grupo A & Perlidae & 3,61 & 12,33 & 17,87 & 17,87 \\
\hline Similitud promedio: $68,98 \%$ & Baetidae & 3,36 & 12,31 & 17,85 & 35,72 \\
\hline \multirow[t]{3}{*}{ Muestras sitios 1 y 8 . } & Chironomidae & 3,35 & 11,7 & 16,96 & 52,68 \\
\hline & Oligochaeta & 3,06 & 11,51 & 16,69 & 69,37 \\
\hline & Dogielinotidae & 3,41 & 9,41 & 13,65 & 83,02 \\
\hline Grupo $B$ & Oligochaeta & 5,51 & 23,41 & 33,24 & 33,24 \\
\hline Similitud promedio: $70,40 \%$ & Dogielinotidae & 4,05 & 16,35 & 23,23 & 56,47 \\
\hline \multirow[t]{2}{*}{ Muestras sitios 2 y 3} & Chironomidae & 3,27 & 13,17 & 18,7 & 75,17 \\
\hline & Perlidae & 2,91 & 10,3 & 14,63 & 89,81 \\
\hline Grupo $C$ & Oligochaeta & 7,41 & 28,39 & 40,84 & 40,84 \\
\hline Similitud promedio: $69,52 \%$ & Chironomidae & 4,61 & 17,21 & 24,76 & 65,6 \\
\hline Muestras sitios 4, 6.2, 6.3, 7.1 y 7.2 & Glossiphoniidae & 2,66 & 9,31 & 13,39 & 78,98 \\
\hline Grupo $D$ & Oligochaeta & 4,67 & 50,73 & 65,19 & 65,19 \\
\hline \multicolumn{6}{|l|}{ Similitud promedio: $77,82 \%$} \\
\hline \multicolumn{6}{|l|}{ Muestras sitios $6.1,6.4,7.3$ y 7.4} \\
\hline Grupo $E$ & Oligochaeta & 7,1 & 38,21 & 39,9 & 39,9 \\
\hline Similitud promedio: $95,76 \%$ & Nematodes & 5,11 & 28,78 & 30,05 & 69,95 \\
\hline Muestras sitios 5 & Ostracodos & 4,96 & 28,78 & 30,05 & 100 \\
\hline
\end{tabular}

Tabla 3: Análisis SIMPER. Principales taxa que contribuyen a la similitud de muestras dentro de cada grupo en verano. $\mathrm{AP}=\mathbf{a b u n d a n c i a}$ promedio (datos transformados); $\mathrm{SP}=$ similitud promedio; \% $\mathrm{C}=$ porcentaje de contribución (\%); \% AC=porcentaje acumulado de contribución.

\begin{tabular}{lccccc}
\hline & Taxa & AP & SP & \%C & \%AC \\
\hline Grupo A & Dogielinotidae & 9,9 & 18,97 & 30,1 & 30,1 \\
Similitud promedio: 63,01\% & Chironomidae & 5,44 & 8,87 & 14,08 & 44,19 \\
Muestras sitios 1, 2 y 8 & Oligochaeta & 5,02 & 7,98 & 12,67 & 56,86 \\
& Baetidae & 5,49 & 6,81 & 10,8 & 67,66 \\
& Elmidae & 3,89 & 6,53 & 10,37 & 78,02 \\
\hline Grupo B & Dogielinotidae & 21 & 31,92 & 47,76 & 47,76 \\
Similitud promedio: 66,84\% & Oligochaeta & 12,98 & 15,24 & 22,8 & 70,56 \\
Muestras sitios 3 y 4 & & & & & \\
\hline Grupo C & Oligochaeta & 37,52 & 39,53 & 57,94 & 57,94 \\
Similitud promedio: $\mathbf{6 8 , 2 2 \%}$ & Chironomidae & 9,82 & 11,19 & 16,39 & 74,33 \\
Muestras sitios 6 y 7 & & & & & \\
\hline Grupo D & Oligochaeta & 8,18 & 44,31 & 63,76 & 63,76 \\
Similitud promedio: 69,51\% & Chironomidae & 2,75 & 15,60 & 22,45 & 86,20 \\
Muestras sitios 5 & & & & & \\
\hline
\end{tabular}

En las Tablas 4 y 5 se pueden observar los valores promedio del índice de diversidad (H'), la abundancia total, el número de taxa (S) y los diferentes índices bióticos calculados para cada sitio de muestreo en las estaciones de primavera y verano. 
Tabla 4: Promedio de los diferentes índices para los sitios muestreados en primavera. EPT (Ephemeroptera, Plecoptera, Trichoptera), EPTCh (EPT, Chironomidae), BMPS (Biological Monitoring Patagonian Streams), ASPT (Average Score Per Taxa), S ( $\mathbf{N}^{0}$ de Taxa), H' (Índice de Shannon-Wiener).

\begin{tabular}{cccccccc}
\hline & EPT & EPTCh & BMPS & ASTP & S & Abundancia & H' $^{\prime}$ \\
\hline 1 & 31,71 & 42,95 & 49,50 & 4,82 & 10,50 & 392,75 & 1,74 \\
2 & 5,92 & 16,91 & 41,75 & 4,19 & 10,00 & 723,50 & 1,14 \\
3 & 8,42 & 13,50 & 28,25 & 4,65 & 6,50 & 484,75 & 0,76 \\
4 & 1,38 & 10,29 & 35,75 & 4,06 & 9,00 & 2665,75 & 0,61 \\
5 & 0,00 & 0,00 & 8,00 & 2,67 & 3,00 & 1696,50 & 0,65 \\
6 & 0,73 & 4,23 & 23,50 & 4,03 & 5,75 & 2177,50 & 0,21 \\
7 & 0,39 & 10,72 & 17,25 & 3,30 & 4,75 & 722,25 & 0,65 \\
8 & 46,01 & 65,34 & 36,33 & 4,73 & 7,67 & 84,67 & 1,75 \\
\hline
\end{tabular}

Tabla 5: Promedio de los diferentes índices para los sitios muestreados en verano. EPT (Ephemeroptera, Plecoptera, Trichoptera), EPTCh (EPT, Chironomidae), BMPS (Biological Monitoring Patagonian Streams), ASPT (Average Score Per Taxa), S ( ${ }^{\circ}$ de Taxa), H' (Índice de Shannon-Wiener).

\begin{tabular}{cccccccc}
\hline & EPT & EPTCh & BMPS & ASTP & S & Abundancia & $\mathbf{H}^{\prime}$ \\
\hline $\mathbf{1}$ & 18,39 & 20,91 & 51,75 & 4,81 & 10,75 & 374,00 & 1,64 \\
$\mathbf{2}$ & 8,87 & 21,72 & 41,75 & 4,66 & 8,75 & 189,25 & 1,36 \\
$\mathbf{3}$ & 3,87 & 12,24 & 39,75 & 4,82 & 8,00 & 246,00 & 1,24 \\
$\mathbf{4}$ & 0,06 & 2,45 & 27,25 & 3,56 & 7,25 & 1083,00 & 1,20 \\
$\mathbf{5}$ & 2,13 & 12,90 & 23,50 & 3,82 & 6,00 & 144,75 & 0,60 \\
$\mathbf{6}$ & 8,19 & 8,69 & 32,75 & 5,45 & 8,50 & 3658,25 & 0,54 \\
$\mathbf{7}$ & 2,31 & 9,50 & 36,25 & 4,29 & 9,25 & 831,25 & 0,52 \\
$\mathbf{8}$ & 21,68 & 45,76 & 42,25 & 4,59 & 9,25 & 173,50 & 1,51 \\
\hline
\end{tabular}

En ambas épocas del año se puede observar que los sitios 1,2 y 8 contienen los valores más altos de cada uno de los índices calculados, mientras que los valores más bajos varían en cada una de las épocas entre los sitios 4, 5, 6 y 7, dependiendo del índice biótico considerado.

Las pruebas de Kruskal-Wallis para determinar si existieron diferencias significativas en los valores de los índices entre sitios de muestreo, arrojaron que los sitios 1,2 y 8 se diferenciaron significativamente de los sitios 5, 6 y 7 en la mayoría de los índices, salvo para el número de taxa $(S)$, que no presentó diferencias significativas entre el sitio 8 y los sitios 5 , 6 у 7 (KW: g.l.= 7; p = 0,0025 para EPT; KW: g.l.= 7; p = 0,0040 para EPTCch; KW: g.l.= 7; $\mathrm{p}=0,0123$ para BMPS; KW: g.l. $=7 ; \mathrm{p}=0,0429$ para ASPT; KW: g.l. $=7 ; \mathrm{p}=0,0083$ para S; $\mathrm{KW}$ : g.l. $=7 ; \mathrm{p}=0,0032$ para $\left.\mathrm{H}^{\prime} ;\right)$. La abundancia fue la única medida que no arrojó diferencias significativas entre las estaciones de muestreo.

El análisis de correlación de Spearman entre los índices calculados y las variables ambientales que presentaron diferencias espaciales significativas, demostró que en primavera el BMPS tuvo correlaciones negativas significativas con la mayoría de las variables (Tabla 6), EPTCh tuvo una correlación negativa significativa con Sólidos Totales, Dureza, DQO y Concentración de Hierro, mientras que $\mathrm{S}$ mostro su correlación significativa con el $\mathrm{pH}$, Sólidos Totales, Dureza y Concentración de Hierro. 
Tabla 6: Coeficientes de correlación de Spearman entre variables e índices calculados para primavera. pH, Cond. (Conductividad), ST (Sólidos Totales), Du (Dureza), DQO (Demanda Química de Oxígeno), y Fe (Concentración de Hierro). * Nivel de significancia p < 0,05.

\begin{tabular}{ccccccc}
\hline & EPT & EPTCh & BMPS & ASPT & S & $\mathbf{H}^{\prime}$ \\
\hline $\mathbf{p H}$ & $-0,67$ & $-0,57$ & $\mathbf{- 0 , 8 6} *$ & $\mathbf{- 0 , 7 4} *$ & $\mathbf{- 0 , 8 8} *$ & $-0,49$ \\
Cond & $-0,55$ & $-0,69$ & $\mathbf{- 0 , 7 9} *$ & $-0,57$ & $-0,71$ & $-0,51$ \\
ST & $-0,54$ & $\mathbf{- 0 , 7 2} *$ & $\mathbf{- 0 , 7 8} *$ & $-0,56$ & $\mathbf{- 0 , 7 1} *$ & $-0,55$ \\
CL & $-0,19$ & $-0,48$ & $-0,36$ & $-0,14$ & $-0,29$ & $-0,26$ \\
Du & $-0,6$ & $\mathbf{- 0 , 7 4} *$ & $\mathbf{- 0 , 8 8} *$ & $-0,62$ & $\mathbf{- 0 , 8 6} *$ & $-0,56$ \\
Turb & $-0,45$ & $-0,62$ & $-0,67$ & $-0,43$ & $-0,57$ & $-0,5$ \\
DQO & $-0,61$ & $\mathbf{- 0 , 8 1} *$ & $\mathbf{- 0 , 7 5} *$ & $-0,57$ & $-0,67$ & $-0,65$ \\
Fe & $-0,6$ & $\mathbf{- 0 , 7 4} *$ & $\mathbf{- 0 , 8 8} *$ & $-0,62$ & $\mathbf{- 0 , 8 6} *$ & $-0,56$ \\
\hline
\end{tabular}

En verano, el índice BMPS mostró correlaciones significativas con la Conductividad, Sólidos Totales, Turbidez y DQO, al igual que S. El índice EPT sólo mostró correlaciones significativas con Sólidos Totales y Turbidez, mientras que el resto de los índices no mostraron correlación significativa con ninguna de las variables ambientales consideradas (Tabla 7).

Tabla 7: Coeficientes de correlación de Spearman entre variables e índices calculados para Verano. pH, Cond. (Conductividad), ST (Sólidos Totales), Cl (Cloruros), Du (Dureza), Turb (Turbidez), DQO (Demanda Química de Oxígeno), y Fe (Concentración de Hierro). * Nivel de significancia $\mathrm{p}<0,05$.

\begin{tabular}{ccccccc}
\hline & EPT & EPTCh & BMPS & ASPT & S & H' \\
\hline $\mathbf{p H}$ & 0 & 0,1 & $-0,31$ & $-0,05$ & $-0,17$ & 0,59 \\
Cond & $-0,71$ & $-0,69$ & $\mathbf{- 0 , 8 6} *$ & $-0,14$ & $\mathbf{- 0 , 7 4} *$ & $-0,64$ \\
ST & $\mathbf{- 0 , 8 4} *$ & $-0,61$ & $\mathbf{- 0 , 8 5 *}$ & $-0,44$ & $\mathbf{- 0 , 8 3} *$ & $-0,56$ \\
Cl & $-0,31$ & $-0,12$ & $-0,57$ & 0,05 & $\mathbf{- 0 , 7 8} *$ & $-0,29$ \\
Du & $-0,6$ & $-0,48$ & $-0,71$ & $-0,02$ & $-0,67$ & $-0,64$ \\
Turb & $\mathbf{- 0 , 8 1} *$ & $-0,57$ & $\mathbf{- 0 , 7 4} *$ & $-0,31$ & $\mathbf{- 0 , 7 3} *$ & $-0,52$ \\
DQO & $-0,69$ & $-0,64$ & $\mathbf{- 0 , 8 1} *$ & 0,02 & $\mathbf{- 0 , 7 5} *$ & $-0,64$ \\
Fe & $-0,64$ & $-0,45$ & $-0,69$ & $-0,14$ & $-0,61$ & $-0,67$ \\
\hline
\end{tabular}

\section{DISCUSIÓN}

Los resultados de los análisis fisicoquímicos del agua indican que existen variaciones espaciales significativas en las variables ambientales asociadas a las fuentes de impacto que existen en el área $\mathrm{El}$ incremento en diferentes parámetros como conductividad, sólidos totales, dureza, turbidez, DQO y concentración de hierro, en particular en el Sitio 5, indicarían un grado de alteración importante, producto de las diferentes actividades antrópicas que se desarrollan en este sector de la cuenca.

Caballero (2009 a y b) detectó que el arroyo San José presenta grados de alteración altos a muy altos en virtud que, sobre este curso de agua desembocan los efluentes pluviales, cloacales e industriales asociados a la localidad de Río Turbio y a la mina operada por la 
empresa YCRT y demás actividades humanas asociadas a ellas. Por otro lado, Udaquiola, Barbieri, Caballero, Grossi y Caliva (2008) mencionan también que en varios trabajos se ha demostrado que la afectación del arroyo San José se puede detectar hasta 70 kilómetros aguas abajo desde su confluencia con el río Turbio. Coincidentemente con estos, la conductividad, uno de los parámetros que aumenta en el Sitio 5, se registra con valores altos hasta el Sitio 3. Este último se emplaza en un sector del río Turbio que transcurre a través de la Estancia Glencross, alrededor de $70 \mathrm{~km}$ de la confluencia del arroyo con el río Turbio, en donde además encontramos presencia de carbonilla y magnetita en el sustrato.

Caballero (2009 a y b) también registra un alto potencial de drenaje ácido de mina (DAM) en los estériles provenientes de las labores de minado y depositados sobre el valle del arroyo San José. Sin embargo, en el presente estudio, el pH en todos los cursos de agua se registró en valores cercanos a neutro. Posiblemente esto se deba a que el alto contenido de carbonato de calcio (en forma de calcita) presente en los estériles esté actuando como buffer, amortiguando el descenso del $\mathrm{pH}$ que podría generar el lixiviado ácido. Asimismo, es común en el paquete sedimentario de la cuenca la presencia de estratos con coquinas y contenido fósil carbonático, por lo cual este podría ser otro factor que morigere la presencia de ácido lixiviado de los estériles.

Por la evidencia recolectada en el área de estudio, podríamos hablar de la posibilidad de drenaje de mina sólo debido a la actividad que se desarrolla en las inmediaciones, pero no se pudo asociar este fenómeno específicamente a un sector particular o acumulación industrial, como lo resalta Bruns (2005). Esto es en parte debido a que las formaciones geológicas portadoras de los mantos de carbón afloran en diversas zonas de la región, exponiéndose en superficie la misma composición mineral que es expuesta también por las actividades de minado. Este autor realizó un trabajo sobre la respuesta de la comunidad de macroinvertebrados a diferentes usos de la tierra en un sistema hídrico impactado por la actividad minera en el noreste de Pensilvania y el sur de Nueva York. El autor encontró que el $\mathrm{pH}$ en la mayoría de los sitios más afectados rondaba el valor neutro, indicando que estos sitios expuestos a condiciones de "drenaje de mina" no manifestaban necesariamente acidificación del medio acuático.

En el presente estudio, la comunidad de macroinvertebrados bentónicos mostró diferencias espaciales significativas entre los diferentes sitios de muestreo. La mayor diversidad se registró en los sitios 1, 2 y 8, mientras que la menor se registró en los sitios 5, 6 y 7 que presentaron mayor abundancia total pero muy pocos taxa. Estos resultados concuerdan con Miserendino et al. (2011) para ríos del norte de Patagonia, quien indica que los principales impactos se detectaron en las áreas urbanas, produciendo significativos cambios en el ecosistema acuático que afectaron a las características físicas del cuerpo de agua, la concentración de nutrientes, las condiciones de hábitats, la calidad de la ribera y también a las comunidades de macroinvertebrados del litoral.

Wright y Brugin (2009) compararon la afectación de la comunidad de macroinvertebrados, producto de su exposición a dos tipos de efluentes, uno minero y otro de aguas residuales urbanas tratadas en ríos del sureste de Australia. Estos autores encontraron que la abundancia de individuos difirió significativamente de los sitios de referencia, siendo significativamente mayor en el sitio inmediatamente aguas abajo del punto de vertido de la planta de efluentes y significativamente menor en el sitio inmediatamente aguas abajo del punto de ingreso de los efluentes mineros. En ambos sitios, observaron además una disminución en la riqueza de familias. Por otro lado, encontraron que las familias Elmidae y Baetidae sufrieron una acentuada disminución en la abundancia en las zonas afectadas por la actividad minera, mientras que en la afectada por la contaminación orgánica ambas familias respondieron con una reducción moderada. En nuestra área de estudio, estas mismas familias presentaron una 
marcada disminución de su abundancia en los sitios 5, 6 y 7, respondiendo de una manera similar a la señalada por los autores para los sitios afectados por la actividad minera.

El comportamiento de la comunidad de macroinvertebrados observado en el presente trabajo, podría estar indicando que en nuestra área de estudio estaría actuando la descarga de la actividad minera conjuntamente con el vertido de efluentes urbanos provenientes de la localidad de Río Turbio, no pudiéndose distinguir en este trabajo el efecto particular de cada fuente de impacto.

Los análisis multivariados realizados demostraron que la comunidad de macroinvertebrados en el Sitio 5 fue significativamente diferente respecto de los sitios 1 y 8 , el primero ubicado aguas abajo y más lejano en el gradiente de impacto y el segundo ubicado aguas arriba del sitio problema y en otra subcuenca. Por otro lado, el Sitio 5 mostró diferencias menos marcadas con la comunidad en los sitios más próximos ubicados aguas abajo en el gradiente de impacto (sitios 4, 6 y 7), con los cuales comparte taxa y características generales de la comunidad. De acuerdo a los análisis multivariados realizados, se podría caracterizar a la comunidad macrobentónica en los diferentes sectores del área de estudio de la siguiente manera:

-En los sitios 1 y 8 la comunidad macrobentónica se caracteriza por su mayor diversidad; se destaca la importante presencia de anfípodos Hyalella sp. e insectos indicadores de buena calidad de agua (Plecoptera, Ephemeroptera y Trichoptera) y la baja abundancia relativa de oligoquetos. Estos sitios podrían corresponderse con un grado de impacto nulo o muy bajo.

-En los sitios 2 y 3 la comunidad macrobentónica se caracteriza por presentar valores intermedios de diversidad; existe una mayor abundancia de oligoquetos y se aprecia una disminución en la abundancia de organismos indicadores de buena calidad de agua, respecto a los sitios 1 y 8 . Estos sitios podrían corresponderse con un grado de impacto bajo.

-En los sitios 4, 6 y 7 la comunidad de macroinvertebrados se caracteriza por tener baja diversidad, mayor abundancia de oligoquetos y quironómidos, y muy baja abundancia o ausencia de organismos indicadores de buena calidad de agua. Estos sitios podrían corresponderse con un impacto moderado.

-En el Sitio 5 la comunidad bentónica se caracteriza por tener el más bajo nivel de diversidad, el menor número de taxa, una amplia dominancia de oligoquetos y la ausencia de especies indicadores de buena calidad de agua. Este sitio se puede considerar el de mayor impacto en el área de estudio.

Los índices bióticos calculados en el presente trabajo respondieron, en general, según lo esperado, encontrándose los valores significativamente más bajos en los sitios más afectados. Como lo señalan Trípole et al. (2005) para el arroyo Carolina en la Provincia de San Luis, la riqueza (S), el índice de Shannon-Wiener y el índice EPT en los sitios problema presentaron valores más bajos que los hallados en los sitios de referencia.

El índice BMPS se correlacionó significativamente con la mayoría de los parámetros fisicoquímicos evaluados, que presentaron cambios significativos a lo largo del gradiente de alteración ambiental.

Esté índice presentó sus valores más bajos en el Sitio 5 en primavera, y en los sitios 5 y 4 en verano, lo que estaría mostrando que en estos sitios el agua presenta una menor calidad respecto de los demás sitios analizados. Por otra parte, los valores más altos de este índice se presentaron en los sitios 1, 2 y 8 en ambas épocas del año, indicando una mejor calidad ambiental en el sector del río aguas arriba del Sitio 5 y en el sector más alejado aguas abajo.

El índice BMPS es relativamente fácil de usar y de bajo costo, y se sugiere su uso para la detección de contaminación incipiente. También se puede utilizar para detectar otros tipos de interferencias provenientes de las crecientes actividades económicas basadas en la explotación 
de los recursos naturales de la Patagonia (regulaciones de ríos, los incendios forestales, la desertificación, la minería) (Miserendino et al. 1999).

\section{CONCLUSIONES}

Se caracterizó la comunidad de macroinvertebrados bentónicos de la cuenca superior del río Gallegos y se propusieron índices bióticos a tener en cuenta para determinar rápidamente la calidad ambiental, generando una línea de base de referencia para contrastar con futuros emprendimientos en la zona.

Se determinó que el Sitio 5 es el que registró el mayor grado de afectación de la zona, en función de las variadas actividades antrópicas que se desarrollan en ese sector del río, sin embargo, no fue posible atribuir de forma concluyente sólo a la actividad minera el impacto observado sobre el recurso hídrico.

Se lograron identificar organismos bentónicos que pueden ser utilizados como indicadores de impacto antrópico en la cuenca superior del río Gallegos, como los oligoquetos e insectos Chironomidae cuya abundancia elevada en la comunidad es indicadora de aguas con un grado de impacto elevado, mientras que la presencia de insectos de las familias Ephemeroptera, Plecoptera y Trichoptera son indicadores de mejor calidad ambiental.

Es posible la utilización de la comunidad de macroinvertbrados bentónicos para la evaluación de la calidad ambiental de los cursos de agua que conforman la cuenca superior del río Gallegos.

Se sugiere la utilización del índice BMPS como índice biótico para la evaluación de la calidad ambiental del recurso hídrico en la cuenca superior del río Gallegos, a partir de haber comprobado su sensibilidad respecto a las alteraciones antrópicas en la zona de estudio.

Se propone la continuación de los trabajos de investigación con el objetivo de ampliar el campo de conocimiento en la materia y ajustar el índice de calidad ambiental propuesto a la compleja realidad productiva y ambiental de la cuenca superior del río Gallegos.

\section{AGRADECIMIENTOS}

Este trabajo se desarrolló en el marco del Proyecto de Investigación PI 29/A299; "Evaluación de la Calidad ambiental Mediante Indicadores en la Patagonia Austral. La extracción de Carbón, la Generación de Energía y el Crecimiento Poblacional Inducido Como Factores de Alteración del Ambiente en la Cuenca Superior del río Gallegos, Santa Cruz". Universidad Nacional de la Patagonia Austral.

\section{BIBLIOGRAFÍA}

ALLAN, J. D. (1995). Stream ecology: structure and function of running waters. Chapman y Hall, Londres, Great Britain. En Alonso y Camargo 2005.

ALONSO, A.; CAMARGO, J.A. (2005). Estado actual y perspectivas en el empleo de la comunidad de macroinvertebrados bentónicos como indicadora del estado ecológico de los ecosistemas fluviales españoles. Asociación Española de Ecosistemas Terrestres. Ecosistemas 14 (3): 87-99. https://doi.org/10.7818/ecos.2017.26-1.05

ANGELIER, E. (2002). Ecología de las aguas corrientes. Editorial Acribia S. A., Zaragoza, España. Citado en Alonso y Camargo 2005.

ARMITAGE, P.D., BLACKBURN, J. H. (1985). Chironomidae in a Pennine stream receiving mine drainage and organic enrichment. Hydrobiologia 121:165-172. Citado en Batty et al 2005. https://doi.org/10.1007/bf00008720 
ASUETA, R., SÚNICO, A., VALlEJOS, J., MARTIN, J. P., FRANCISCOVIC, K., GUGLIELMINETTI, G; AGUAS, P. S. (2013). Macroinvertebrados de la Cuenca del río Gallegos, Santa Cruz. Su Asociación con la Calidad Ambiental. Ponencia en $4^{\circ}$ Congreso de Ciencias Ambientales COPIME 2013.

BATTY, L.C.; ATKIN, L.; MANNING, D.A.C. (2005). Assessment of the ecological potential of mine-water treatment wetlands using a baseline survey of macroinvertebrate communities. Environmental Pollution 138:412-419. https://doi.org/10.1016/i.envpol.2005.04.022

BANKS, D., YOUNGER, P. L., ARNESEN, R. T., IVERSEN, E. R., BANKS, S. B. (1997). Mine water chemistry: the good, the bad and the ugly. Environmental Geology 32:157-174. Citado en Batty et al 2005. https://doi.org/10.1007/s002540050204

BONADA, N., PRAT, N., RESH V. H. y STARTZNER, B. (2006). Developments in aquatic insect biomonitoring: A comparative analysis of recent approaches. Annu. Rev. Entomol. 55:495-523. https://doi.org/10.1146/annurev.ento.51.110104.151124

BRANCO, S. (1984). Limnología Sanitaria, estudio de la polución de las aguas continentales. Editorial Secretaría general de la Organización de los Estados Americanos. 120pp. Citado en Gamboa et al. 2008. https://doi.org/10.18356/9e6a0dc8-es

BRUNS, D. A. (2005). Macroinvertebrate response to land cover, habitat, and water chemistry in a mining-impacted river ecosystem: A GIS watershed analysis. Dübendorf. Aquat. Sci. 67:403 - 423. https://doi.org/10.1007/s00027-005-0792-3

CABALlERO, A. (2009 a). Evaluación Preliminar De Depósitos De Estériles Y sedimentos Provenientes De La Actividad Carbonífera En Río Turbio. ICT-UNPA-3-2009 Universidad Nacional de la Patagonia Austral. Unidad Académica Río Turbio. https://doi.org/10.22305/ict-unpa.v1i1.11

CABALleRO, A. (2009 b). Valoración Del Impacto De Efluentes Minero Industriales Del Carbón En El Arroyo San José De Río Turbio En Santa Cruz. ICT-UNPA-4-2009 Universidad Nacional de la Patagonia Austral. Unidad Académica Río Turbio. https://doi.org/10.22305/ict-unpa.v1i1.12

CABALlERO, J. O. (2000). Hidrografía y Recursos Hídricos. El Gran Libro de la Provincia de Santa Cruz. Milenio. 116 - 139.

CARPENTER, S. R., FISER, S. G., GRIMM N. B. y KITCHELL J. F. (1992). Global Change and freshwater ecosystems. Annual Review of Ecology and Sistematics. 23:119-139. Citado en Bruns 2005. https://doi.org/10.1146/annurev.es.23.110192.001003

CLARKE, K. R., WARWICK, R. M. (2001). Change in marine communities: an approach to statistical analyses and interpretation, PRIMER-E, Plymouth.

CONFEDERACIÓN HIDROLÓGICA DEL EBRO (2005). Protocolo de muestreo y análisis de invertebrados bentónicos. 47pp.

ESTEVES, J. L., GIL, M., Harvey, M. (1996). Informes Técnicos del Plan de Manejo Integrado de la Zona Costera Patagónica (Puerto Madryn, Argentina) № 12.

DOMÍNGUEZ, E. y FERNÁNDEZ, H. R. (2009). Macroinvertebrados Bentónicos Sudamericanos-Sistemática y Biología (eds.) Fundación Miguel Lillo, Tucumán, Argentina. https://doi.org/10.1603/008.103.0401

FERNÁNDEZ, H. R. y DOMÍNGUEZ, E. (2001). Guía para la determinación de los artrópodos bentónicos sudamericanos. Universidad Nacional de Tucumán. 282 pp.

GAMBOA, M., REYES, R., ARRIVILLAGA, J. (2008). Macroinvertebrados bentónicos como bioindicadores de salud ambiental. Bol Mal Salud Amb v.48 n.2 Maracay dic. 2008 . 
GRAY, N. F., DENLEY, E. (2008). Comparison of benthic macroinvertebrate indices for the assessment of the impact of acid mine drainage on an Irish river below an abandoned $\mathrm{Cu}-\mathrm{S}$ mine. Environmental Pollution 155.31 - 40. https://doi.org/10.1016/j.envpol.2007.11.002

HAUER, F. R. y RESH, V. H. (1996). Benthic Macroinvertebrates. Pp. 339-369 in Hauer F.R. y G.A. Lamberti (ed): Methods in Stream Ecology. Academy Press, New York. https://doi.org/10.1016/b978-012332908-0.50028-0

JARVIS, A. P., YOUNGER, P. L. (1997). Dominating chemical factors in mine waterinduced impoverishment of the invertebrate fauna of two streams in the Durham coalfield, UK. Chemistry and Ecology.13:249-270. https://doi.org/10.1080/02757549708035531

MALMQVIST, B., HOFFSTEN, P. O. (1999). Influence of drainage from old mine deposits of benthic macroinvertebrate communities in central Swedish streams. Water Research 33:2415-2423. Citado en Batty et al. 2005. https://doi.org/10.1016/s00431354(98)00462-X

MANZANO, M., CUSTODIO, E., PONCELA, R. (1991). Contribución de la hidrogeoquímica al conocimiento de la hidrodinámica de los acuíferos del área de Doña Ana. III Simposio Sobre el Agua en Andalucia. Córdoba-Spain.: SIAGA, 1991:475-86. Citado en Manzano et al. 1999. https://doi.org/10.1127/phyto/19/1991/547

MANZANO, M., AYORA, C., DOMENECH, C., NAVARRETE, P., GARRALON, A., TURRERO, M. J. (1999). The impact of the Aznalcóllar mine tailing spill on groundwater. The Science of the Total Environment 242:189-209. https://doi.org/10.1016/s0048-9697(99)00384-8

MARTIN, J. P., SAR, A., FERNÁNDEZ, R., CAMINOS, C., PEREYRA GINESTAR, B., MOSCARDI, C. y GASPARI, G. (2014). Diversidad de macroinvertebrados bentónicos y aplicación de índices bióticos de calidad ambiental en el río Santa Cruz (Patagonia austral, Argentina). Memorias del II Congreso Internacional de Hidrología de Llanuras, Universidad Nacional del Litoral, ISBN 978-987-692-039-1. https://doi.org/10.30972/fac.310650

MISERENDINO, M. L., y PIZZOLON, L. (1992). Un índice biótico de calidad de aguas corrientes para la región andino-patagónica. Resumo do II Congreso Latinoamericano de Ecología. Pp 39-40.

MISERENDINO, M. L., y PIZZOLON, L. (1999). Rapid assessment of river water quality using macroinvertebratess: a family level biotic index for the Patagonic Andean zone. Acta Limnol. Bras. 11:137-148.

MISERENDINO, M. L. (2000). Distribución y variación estacional de plecoptera en un sistema fluvial de cordillera Patagónica. Revista de la Sociedad Entomológica de Argentina 59; 149-160.

MISERENDINO, M. L. (2001). Macroinvertebrate assemblages in Andean Patagonian rivers and streams: environmental relationships. Hidrobiología. 444: 147-158.

MISERENDINO, M. L., BRAND, C., y PRINIZIO, C. Y. (2008). Assessing Urban Impacts on Water Quality, Benthic Communities and Fish in Streams of the Andes Mountains, Patagonia (Argentina). Water, Air, and Soil Pollution. 91-110. https://doi.org/10.1007/s11270-008-9701-4

MISERENDINO, M. L. (2009). Effects of flow regulation, basin characteristics and land-use on macroinvertebrate communities in a large arid patagonian river. Biodiversity y Conservation. 1921-1943. https://doi.org/10.1007/s10531-008-9565-3 
MISERENDINO M. L. y BRAND, C. (2009). Environmental effects of urbanization on streams and rivers in Paragonia (Argentina): The use of macroinvertebrates in monitoring. In Ed. Justin Daniels. Advancces in Environmental Research Volume 6. Chapter 5. NOVA. New York. 183 - 220 pp. ISBN: 978-1-61728-163-1.

NIEMI, G. J. y MCDONALD M. E. (2004). Application of ecological indicators. Annu.Rev. Ecol. Evol. Syst. 35:89-111. Citado en Bonada et al. 2006. https://doi.org/10.1146/annurev.ecolsys.35.112202.130132

PRAT, N. y WARD, J. V. (1994). The tamed river. En Limnology now: a paradigm of planetary problems (ed Margalef, R.), pp. 219-236, Elsevier Science. Citado en Alonso y Camargo 2005.

PRAT, N., RÍOS, B., ACOSTA, R. y RIERADEVALL, M. (2008). Los Macroinvertebrados como Indicadores de Calidad de Aguas. Macroinvertebrados Bentónicos Sudamericanos. Capítulo 20. Fundación Miguel Lillo. https://doi.org/10.11606/d.6.1999.tde-15022007-145012

RODRIGUES CAPITULO, A. R., TANGORRA, M. y OCÓN, C. (2001). Use of benthic macroinvertebrates to assess the biological status of Pampean streams in Argentina. Aquatic ecology. 35: 109-119.

SEGNINI, S. (2003). El uso de los macroinvertebrados bentónicos como indicadores de la condición ecológica de los cuerpos de agua corriente. Sociedad Venezolana de Ecología. ECOTROPICOS 16(2):45-63. https://doi.org/10.11606/d.18.2014.tde02022015-103604

SUÁREZ, M. L., VIDAL-ABARCA, M. R., SÁNCHEZ-MONTOYA, M. M., ALBATERCEDOR, J., ÁlVAREZ, M., AVILÉS, J., BONADA, N., CASAS, J., JÁIMEZCUÉllar, P., MUNNÉ, A., PARDO, I., PRAT, N., RIERADEVALL, M., SALINAS, M. J., TORO, M. y VIVAS, S. (2002). Las riberas de los ríos mediterráneos y su calidad: el uso del índice QBR. Limnetica 21: 135-148. Citado en Alonso y Camargo 2005.

TAGLIAFERRO, M., MISERENDINO, M. L., LIBEROFF, A., QUIROGA, A. y PASCUAL, M. (2013). Dams in the last large free-flowing rivers of Patagonia, the Santa Cruz River, environmental features, and macroinvertebrate community. Limnologica 43 (2013). Pp 500-509. https://doi.org/10.1016/j.limno.2013.04.002

TORO, M., ROBLES, S., AVILÉS, J., NUÑO, C., VIVAS, S., BONADA, N., PRAT, N., ALBA-TERCEDOR, J., CASAS, J., Guerrero, C., Jáimez-Cuéllar, P., Moreno, J. L., Moyá, G., Ramon, G., Suárez, M. L., VIDAL-ABARCA, M. R., ÁLVAREZ, M. y PARDO, I. (2002). Calidad de las aguas de los ríos mediterráneos del proyecto GUADALMED. Características fisicoquímicas. Limnetica 21: 63-75. Citado en Alonso y Camargo 2005.

TRIPOLE, E. S., CORIGLIANO, M. C. (2005). Acid stress evaluation using multimetric índices in the Carolina stream (San Luis - Argentina), Acta Limnol. Bras. 17(1), 101114.

UDAQUIOLA, S., BARBIERI, V., CABALlERO, A., GROSSI, M y CALIVA, H. (2008). Valoración Cuantitativa del Impacto Minero Industrial del Carbón en un Curso de Agua. Cado del Arroyo San José de Río Turbio. Santa Cruz. Informe Final Proyecto de Investigación. https://doi.org/10.22305/ict-unpa.v1i1.12

VAN HUYSSTEEN, E. (1998): Reseña del Drenaje Ácido de Minas en el Contexto de una Rehabilitación de un Emplazamiento Minero. Lineas de Base. Un Manual de Referencias. Primera Edición CANMET/MMSL - INTEMIN.

WELCH, E. B. y LINDELL, T. (1992). Ecological effects of wastewater: applied limnology and pollutant effects. $2^{\text {nd }}$ ed. E y FN Spon. London. 418p. Citado en Pizzolon y Miserendino, 2001. 
WETZEL, R. G. (2001). Limnology. Lake and River Ecosystems. Academic Press, Third Edition. Citado en Alonso y Camargo 2005.

WRIGHT, I. A. y BRUGIN, S. (2009). Comparison of Sewage and Coal-Mine Wastes on Stream Macroinvertebrates Within an Otherwise Clean Upland Catchment, Southeastern Australia. Water, Air, and Soil Pollution. 204(1-4), 227-241. https://doi.org/10.1007/s11270-009-0040-x

YOUNGER, P. L. (1997). Minewater treatment using wetlands. Proceedings of a National Conference, University of Newcastle, UK. London Chartered Institute of Water and Environmental Management, pp. 65-81. Citado en Batty et al. 2005.

ZOCOLA E. P. (1973). Río Turbio. Gesta del Carbón Argentina. 1943 - 1973. Ministerio de Economía, Secretaría de Estado de la Energía. Yacimientos Carboníferos Fiscales. Bs. As. Pp. 111. 